\title{
FUNÇÕES DE AFILAMENTO PARA Pinus elliottii Engelm. PROVENIENTE DE CONDUÇÃO DE REGENERAÇÃO NATURAL
}

\author{
STEM TAPER FUNCTION FOR Pinus elliottii Engelm. FROM CONDUCITION OF NATURAL \\ REGENERATION
}

\author{
Samuel Alves da Silva ${ }^{1}$, Linamara Smaniotto Ferrari ${ }^{2}$, Diego Moreira ${ }^{3}$, \\ Luciano Rodrigo Lanssanova ${ }^{4}$, Roni Djeison Ansolin ${ }^{5}$, Lucas Dalmolin Ciarnoschi ${ }^{6}$, \\ Nelson Yoshihiro Nakajima \\ 1,2, 3, 5,6,7 Universidade Federal do Paraná, Curitiba, Paraná, Brasil-ssilva.alves@yahoo.com.br, \\ linamaraferrari@gmail.com,diego.moreira@madem.com.br,roni_ansolin@yahoo.com.br, \\ ciarnoschifloresta@gmail.com \&nelson.nakajima@ufpr.br \\ ${ }^{4}$ Instituto Federal de Ciência, Tecnologia e Educação de Mato Grosso, Juína, Mato Grosso, Brasil - \\ luciano.lanssanova@jna.ifmt.edu.br
}

RESUMO

A condução de florestas por regeneração natural pode apresentar perfis de fuste diferentes em relação à indivíduos provenientes de plantios por mudas ou semeadura. Desta forma, o objetivo desse trabalho foi ajustar modelos de funções de afilamento para povoamentos da espécie Pinus elliottii Engelm. proveniente de condução de regeneração natural, afim de identificar o que melhor se ajusta e verificar se a procedência dos indivíduos afeta negativamente os indicadores estatísticos de regressão. Para isso, foram ajustados os modelos de função de afilamento propostos por Kozak et al., Max \& Burkhart, Schöepfer e Hradetzky para um banco de dados de 289 árvores cubadas pelo método de Smalian, das quais, 40 amostras foram utilizadas para validar a melhor equação. A avaliação das equações testadas foi realizada por meio do coeficiente de determinação ajustado $\left(R_{a j}^{2}\right)$, erro padrão da estimativa em percentagem (Syx \%), análise gráfica dos resíduos em porcentagem e média dos desvios (MD). Os modelos ajustados apresentaram resultados estatísticos satisfatórios. Todas as equações ajustadas apresentaram resultados muito semelhantes para $R_{a j .}^{2}$, que variou entre 0,9630 e 0,9807, para o Syx \%, os valores variaram um pouco mais, de 9,0785 a 6,5647, e para MD de $-0,1500$ a 0,0858. O modelo proposto por Max \& Burkhart apresentou os melhores resultados para a estimativa dos diâmetros ao longo do fuste, apresentando o maior $R_{a j \text {. }}^{2}$ o menor Syx \% e MD de 0,0858 . Com os resultados apresentados podemos observar que a aplicação de modelos de afiamento para povoamentos de Pinus elliottii oriundos de regeneração natural é viável.

PALAVRAS-CHAVE: Modelo de perfil, Perfil do fuste, Sortimento.

ABSTRACT

The management of forests by natural regeneration may have different bole profiles of individuals from plantings by seedlings and sowing. In this way, the objective of this work was to adjust models of tapering functions for stands of Pinus elliottii Engelm. from natural regeneration conduction, in order to identify the best fit and to verify if the origin of the individuals negatively affects the statistical regression indicators. In order to do this, the models of the tapering function proposed by Kozak et al., Max \& Burkhart, Schöepfer and Hradetzky were adjusted for a database of 289 trees cubed by the Smalian method, of which 40 samples were used to validate the best equation adjusted. The evaluation of the equations tested was performed using the adjusted coefficient of determination $\left(R_{a d j}^{2}\right)$, standard error estimate in percentage $\left(S_{y x} \%\right)$, graphic analysis of residuals in percentage and average of the deviations (AD). The adjusted models presented satisfactory statistical results. All adjusted equations presented very similar results for $R_{a d j,}^{2}$, which ranged from 0.9630 to 0.9807 , for the $S_{y x} \%$, values ranged slightly from 9.0785 to 6.5647 , and for AD of -0.1500 to 0.0858 . The model proposed by Max \& Burkhart presented the best results for the estimation of the diameters along the bole, presenting the highest $R_{a d j}^{2}$, the lowest $S_{y x} \%$ and AD of 0.0858 . With the results presented, we can observe that the application of tapering models for stands of Pinus elliottii from natural regeneration is feasible. 


\section{INTRODUÇÃO}

A quantificação do potencial madeireiro, realizada a partir de informações provenientes de inventários florestais é uma das atividades mais importantes do planejamento da produção florestal (NAKAJIMA et al., 2011).

Com base nos dados coletados no inventário é possível determinar o volume do povoamento através de diferentes métodos, dentre eles, a função de forma, também conhecida como função de afilamento (SOUZA et al., 2008) que possibilita descrever matematicamente o perfil longitudinal de um fuste e determinar o volume de madeira por meio da integral dessa função, entre quaisquer pontos ao longo do fuste (MENDONÇA et al., 2007), além de permitirem, também, a estimativa do volume de cada produto, a partir de um diâmetro mínimo pré-definido e dessa maneira, permitindo a otimização do uso da madeira (HORLE et al., 2010).

A estimativa acurada de diferentes sortimentos nos plantios florestais demanda pesquisas relacionadas à modelagem do volume e forma das árvores no intuito de se obter uma informação segura e precisa do volume dos multiprodutos advindos da floresta, como em povoamentos do gênero Pinus (MÔRA et al., 2014; MACFARLANE \& WEISKITTEL, 2016). Nessa perspectiva, faz-se necessário o emprego de técnicas mais sofisticadas de modelagem como o uso de funções de afilamento (CARVALHO et al., 2014).

Devido à falta de conhecimento do crescimento e comportamento das espécies nativas aliadas a importação de tecnologia para a produção de celulose e papel, promove-se o uso de espécies exóticas em plantações florestais, em sua maioria pertencentes aos gêneros Pinus e Eucalyptus, que representam a principal fonte de matéria prima do setor florestal brasileiro (YOSHITANI JUNIOR et al., 2012; KOHLER et al., 2013).

A maior concentração de plantios do gênero Pinus está localizada na região sul do Brasil, onde seu cultivo por meio de práticas adequadas de manejo, promove o desenvolvimento social e econômico, além de contribuir para a redução da exploração de florestas nativas ameaçadas (VASQUEZ et al., 2007).

Segundo Ferrari et al. (2017), em determinadas regiões do país, por ser considerada nociva à determinados ecossistemas, a atividade silvicultural com espécies exóticas sofre restrições que podem chegar a proibição do plantio em alguns casos. Sendo assim, como é o caso do cultivo da espécie Pinus elliottii Engelm., em alguns locais no estado do Rio Grande do Sul, a condução da regeneração natural dessas espécies é uma alternativa para a manutenção da atividade silvicultural nesses locais (FERRARI et al., 2017).

Devido ao fato de serem frutos de condução de regeneração, esses indivíduos podem apresentar um perfil diferente dos provenientes de plantios por mudas e semeadura (FOX et al., 2007).

Por não ser uma prática muito difundida no país, não existem muitas informações sobre o sortimento e perfil do fuste de indivíduos provenientes de condução da regeneração natural para fins comerciais. Dessa forma, o objetivo desse trabalho foi ajustar modelos de funções de afilamento para a espécie Pinus elliottii proveniente de condução de regeneração natural, afim de identificar o que melhor se ajusta ao povoamento e verificar se a procedência dos indivíduos afeta negativamente os indicadores estatísticos da regressão.

\section{MATERIAL E MÉTODOS}

\section{Caracterização da área de estudo}

Os dados utilizados neste estudo foram obtidos de um inventário florestal realizado em áreas de regeneração natural de Pinus elliottii com idade variando entre 4 a 20 anos e espaçamentos não definidos. Para condução, entre - $4^{\circ}$ e $6^{\circ}$ anos da regeneração espontânea, foram realizadas as roçadas e seleção dos indivíduos fenotipicamente mais promissores, mantendo 1.500 árvores por hectare. Os povoamentos florestais estão localizados no litoral médio do estado do Rio Grande do Sul, no município de Mostardas - RS, com latitude $31^{\circ} 06^{\prime}$ $1.44^{\prime \prime}$ e Longitude $50^{\circ} 54^{\prime} 54^{\prime \prime}$ e altitude de $17 \mathrm{~m}$ acima do nível do mar.

Conforme a classificação de Köppen atualizado por Álvares et al. (2013), o clima predominante da região é o subtropical de verões quentes ( $\mathrm{Cfa}$ ) com temperatura média anual de 21 으 e pluviosidade média anual de 1.450 $\mathrm{mm}$, os solos da região são classificados como plintossolos álicos, na região de restinga litorânea, planossolos solódicos nas planícies alta e média e areias quartzosas hidromórficas húmidas na planície baixa (MARTIN, 2017; GONZATTI et al., 2014).

\section{Banco de dados}

Foram cubadas 289 árvores, selecionadas com base na distribuição diamétrica do povoamento (Tabela 1). 0 método de cubagem adotado foi o de Smalian, com mensuração dos diâmetros com casca, nas alturas 
absolutas no fuste, a 0,15 - 0,5 - 1,0-1,3 - 1,7 - 2,5 metros, após esta padronizou-se a tomada do diâmetro a cada dois metros, até o ápice do fuste.

Tabela 1. Definição da suficiência amostral para os dados de Pinus sp. oriundos de regeneração natural em Mostardas-RS

\begin{tabular}{cccccc}
\hline Classe & $\begin{array}{c}\text { Intervalo } \\
(\mathbf{c m})\end{array}$ & $\begin{array}{c}\boldsymbol{t} \text { tab. } \\
(\mathbf{9 5 \%})\end{array}$ & CV (\%) & $\begin{array}{c}\text { Amostra } \\
\text { (n árvores) }\end{array}$ & $\begin{array}{c}\text { Suficiência } \\
\text { Amostral }\end{array}$ \\
\hline I & $4 \leq 7$ & 2,07 & 16,08 & 23 & 11 \\
II & $7 \leq 10$ & 2,03 & 10,64 & 38 & 5 \\
III & $10 \leq 13$ & 2,01 & 7,70 & 48 & 2 \\
IV & $13 \leq 16$ & 2,00 & 7,77 & 56 & 2 \\
V & $16 \leq 19$ & 2,01 & 6,94 & 46 & 2 \\
VI & $19 \leq 22$ & 2,02 & 6,41 & 41 & 2 \\
VII & $22 \leq 25$ & 2,06 & 5,95 & 25 & 2 \\
VIII & $25 \leq 28$ & 2,20 & 5,08 & 12 & 2 \\
\hline
\end{tabular}

Em que: $t$ tab.= Valor de $t$ tabelado a $95 \%$ de probabilidade, $\mathrm{e}$ $\mathrm{CV}=$ Coeficiente de variação.

\section{Processamento dos dados}

Para o estudo foram ajustados os modelos de afilamento de Kozak et al. (1969), Max \& Burkhart (1976), Schöepfer (1966) e Hradetzky (1976), representados pelas expressões (1), (2), (3) e (4), respectivamente. Todos os modelos foram ajustados pelo método dos mínimos quadrados.

Modelo de Kozak et al. (1969):

$$
\left(d_{i} / d\right)^{2}=\beta_{0}+\beta_{1}\left(h_{i} / h_{t}\right)+\beta_{2}\left(h_{i} / h_{t}\right)^{2}+\varepsilon_{i}
$$

Modelo de Max \& Burkhart (1976):

$$
\begin{aligned}
d_{i}=d\left[\beta_{1}\left(\left(h_{i} / h_{t}\right)-1\right)+\beta_{2}\left(\left(h_{i} / h_{t}\right)^{2}-1\right)\right. \\
+\beta_{3}\left(a_{1}-\left(h_{i} / h_{t}\right)\right)^{2} I_{1} \\
\left.+\beta_{4}\left(a_{2}-\left(h_{i} / h_{t}\right)\right)^{2} I_{2}\right]^{0,5}+\varepsilon_{i}
\end{aligned}
$$

Modelo de Schöepfer (1966):

$$
\begin{array}{r}
d_{i} / d=\beta_{0}+\beta_{1}\left(h_{i} / h_{t}\right)+\beta_{2}\left(h i / h_{t}\right)^{2}+\beta_{3}\left(h_{i} / h_{t}\right)^{3} \\
+\beta_{4}\left(h_{i} / h_{t}\right)^{4}+\beta_{5}\left(h_{i} / h_{t}\right)^{5}+\varepsilon_{i}
\end{array}
$$

Modelo de Hradetzky (1976):

$$
\begin{gathered}
d_{i} / d=\beta_{0}+\beta_{1}\left(h_{i} / h_{t}\right)^{p 1}+\beta_{2}\left(h_{i} / h_{t}\right)^{p 2}+\cdots \\
+\beta_{n}\left(h_{i} / h_{t}\right)^{p n}+\varepsilon_{i}
\end{gathered}
$$

Em que: $d_{i}=$ Diâmetro $i$ ao longo do fuste da árvore $(\mathrm{cm}), d$ = Diâmetro a altura do Peito, 1,3 $\mathrm{m}$ do solo $(\mathrm{cm})$, $h_{i}=$ Altura $i$ ao longo do fuste da árvore $(\mathrm{m}), h_{t}=$ Altura total do fuste da árvore $(\mathrm{m}), \varepsilon_{i}=$ Erro aleatório, $\beta_{n}=$ Coeficientes estimados, $p n=$ Potências a serem selecionadas para compor o Polinômio de Potências Fracionárias, $a_{\mathrm{i}}=$ Pontos de ligação dos polinômios, $\mathrm{l}_{\mathrm{i}}: \mathrm{l}_{\mathrm{i}}:=1$ se $h_{\mathrm{i}} / \mathrm{h} \leq \mathrm{a}_{\mathrm{i}} ; \mathrm{l}_{\mathrm{i}}:=0$ se $\mathrm{h}_{\mathrm{i}} / \mathrm{h}$ $\geq a_{\text {i }}$.

Para seleção das potências do modelo de Hradetzky, utilizou-se o método Stepwise, para inclusão ou não de uma variável, sendo testadas as potências: 0,$005 ; 0,09$; 0,$08 ; 0,07 ; 0,06 ; 0,05 ; 0,04 ; 0,03 ; 0,02 ; 0,01 ; 0,9 ; 0,8 ; 0,7$; 0,$6 ; 0,5 ; 0,4 ; 0,3 ; 0,2 ; 0,1 ; 1 ; 2 ; 3 ; 4 ; 5 ; 6 ; 7 ; 8 ; 9 ; 10 ; 15 ; 20$; e, 25 , as quais foram consideradas ao nível de significância de $5 \%(\alpha \leq 0,05)$, no teste " $F$ " parcial de cada coeficiente do modelo para a entrada ou saída de uma variável, o método de ajuste foi pelos mínimos quadrados ordinários (MQO) e as potências selecionadas foram 0,$005 ; 0,03 ; 0,2$; $1 ; 2 ; 3$ e 6.

\section{Critérios de seleção da melhor função}

A seleção da melhor equação foi baseada de acordo com as estatísticas de ajuste e precisão, sendo estas o coeficiente de determinação ajustado $\left(R_{a j}^{2}\right)$, o erro padrão da estimativa em percentagem ( $S_{y x} \%$ ), análise gráfica dos resíduos em porcentagem e média dos desvios, que ajudam a definir a aplicabilidade dos modelos por meio da distribuição dos erros ao longo da amplitude de variação das variáveis.

Das 289 árvores que compunham o conjunto total de dados, foram utilizadas 40 amostras independentes para validar a equação selecionada, verificando-se que nenhuma classe diamétrica ficasse sem representação. Segundo Kohler et al. (2013), por meio da validação mostra como uma equação ajustada para um determinado grupo de dados comporta-se na estimativa de dados independentes daqueles utilizados no ajuste. Para tal, foi empregado o teste de Qui-quadrado $\left(\chi^{2}\right)$, a $5 \%$ de probabilidade, para avaliar a existência de diferença estatística.

\section{RESULTADOS}

Os parâmetros estimados para os quatro modelos testados para o conjunto total dos dados são apresentados na Tabela 2, com as respectivas médias dos desvios, erros padrão da estimativa $\mathrm{S}_{\mathrm{yx}} \%$ e $R_{a j \text {. }}^{2}$. 
Tabela 2. Resultados dos ajustes das funções de afilamento para diâmetro com casca de Pinus elliottii

\begin{tabular}{|c|c|c|c|c|c|}
\hline Modelo & \multicolumn{2}{|c|}{ Coeficientes } & $M D$ & $\mathrm{~S}_{\mathrm{yx}}(\%)$ & $R_{a j}^{2}$ \\
\hline \multirow{3}{*}{ (1) } & $b_{0}$ & 1,3465 & \multirow{3}{*}{0,0000} & \multirow{3}{*}{9,0785} & \multirow{3}{*}{0,9630} \\
\hline & $B_{1}$ & $-2,1334$ & & & \\
\hline & 82 & 0,8447 & & & \\
\hline \multirow{6}{*}{ (2) } & $\alpha 1$ & 0,1000 & \multirow{6}{*}{0,0858} & \multirow{6}{*}{6,5647} & \multirow{6}{*}{0,9807} \\
\hline & $\alpha 2$ & 0,5567 & & & \\
\hline & $B_{1}$ & $-2,7567$ & & & \\
\hline & $B_{2}$ & 1,1424 & & & \\
\hline & $B_{3}$ & 56,9042 & & & \\
\hline & $B_{4}$ & $-1,7191$ & & & \\
\hline \multirow{6}{*}{ (3) } & $B_{0}$ & 1,2693 & \multirow{6}{*}{$-0,1500$} & \multirow{6}{*}{7,1161} & \multirow{6}{*}{0,9773} \\
\hline & $B_{1}$ & $-3,8755$ & & & \\
\hline & $B_{2}$ & 17,6407 & & & \\
\hline & $B_{3}$ & $-40,9759$ & & & \\
\hline & $B_{4}$ & 40,9759 & & & \\
\hline & $B_{5}$ & $-15,2071$ & & & \\
\hline \multirow{8}{*}{ (4) } & $B_{0}$ & $-30661,06$ & \multirow{8}{*}{$-0,1396$} & \multirow{8}{*}{6,7763} & \multirow{8}{*}{0,9794} \\
\hline & $B_{1}$ & 38529,1305 & & & \\
\hline & $B_{2}$ & $-8172,1305$ & & & \\
\hline & $B_{3}$ & 322,7783 & & & \\
\hline & $B_{4}$ & $-27,1444$ & & & \\
\hline & $B_{5}$ & 15,1153 & & & \\
\hline & $B_{6}$ & $-6,7825$ & & & \\
\hline & $B_{7}$ & 0,7021 & & & \\
\hline
\end{tabular}

Em que: $\beta_{n}=$ Coeficientes estimados; $M D=$ média dos desvios; $\left(\mathrm{S}_{\mathrm{yx}}\right.$ $\%)$ = Erro padrão da estimativa em percentagem; $R_{a j .}^{2}=$ Coeficiente de determinação ajustado.

Os modelos testados apresentaram boas estatísticas de ajuste. Todas as equações ajustadas apresentaram resultados muito semelhantes, para o $R_{a j .}^{2}$, no entanto, as equações de Hradetzky e Max \& Burkhart apresentam os menores valores para o $\mathrm{S}_{\mathrm{yx}} \%$. Contudo, o modelo de Max \& Burkhart apresentou-se levemente superior ao modelo de Hradetzky. Na Figura 1 é apresentada a análise gráfica dos resíduos.

Observa-se que todos os modelos ajustados apresentaram dispersão residual com alta amplitude que se agravam nos diâmetros menores, chegando a apresentar tendenciosidades. O que demonstra que os modelos ajustados apresentam maior dificuldade para estimar os diâmetros menores.

Com base nos resultados obtidos, é possível afirmar que todos os modelos ajustados apresentaram resultados satisfatórios e poderiam ser utilizadas na obtenção das estimativas dos diâmetros ao longo do tronco das árvores, porém, o modelo o modelo proposto por Max \& Burkhart, além de apresentar uma pequena superioridade no $S_{y x} \%$, também apresentou maior homogeneidade na distribuição de resíduos.
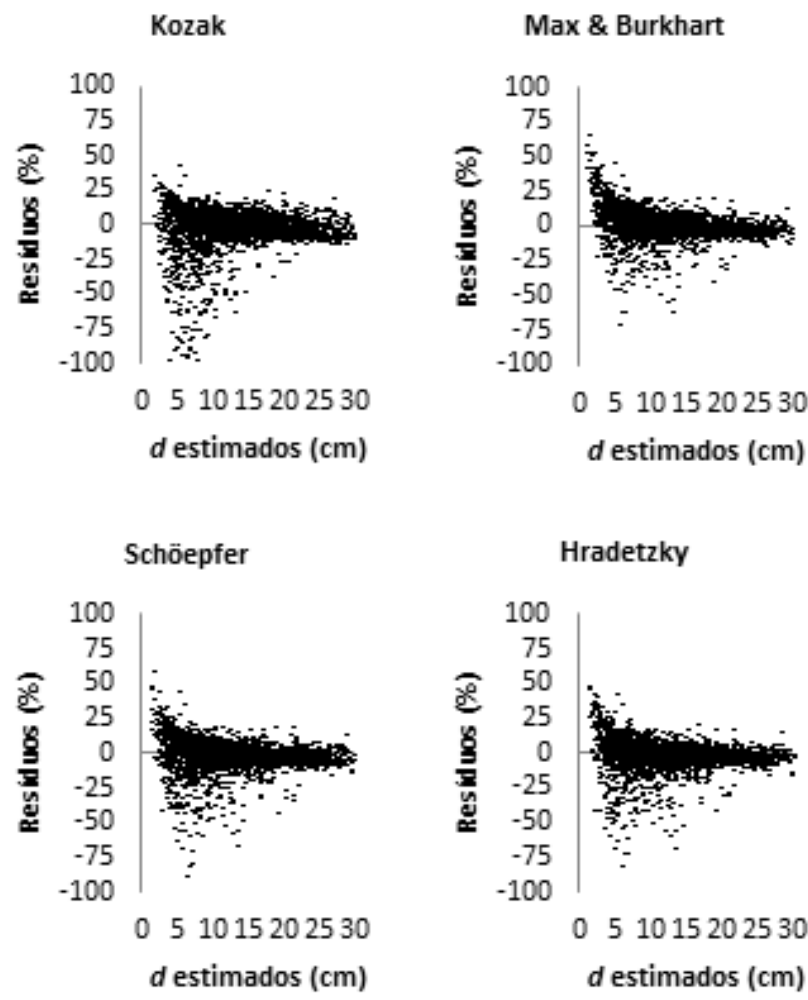

Figura 1. Distribuição dos Resíduos em função dos diâmetros estimados para o conjunto total de dados para os modelos testados para Pinus elliottii.

Na Figura 2 é apresentado o perfil médio do fuste das árvores presentes no banco de dados utilizado neste estudo para as quatro equações ajustadas, se observa que os perfis estimados pelos modelos de Max \& Burkhart, Schöepfer e Hradetzky foram semelhantes. O perfil estimado pelo modelo de Max \& Burkhart apresentou uma linha convexa com relação ao eixo das abscissas, da base até cerca de $10 \%$ da altura total do fuste, onde exibe um ponto de inflexão.

Embora tenha apresentado estatísticas de precisão satisfatória, a linha média de ajuste da equação, que representa o perfil do fuste obtido pelo o modelo de Kozak, se mostrou a de menor eficiência na estimativa dos diâmetros. O perfil obtido pelo modelo de Hradetzky, embora tenha apresentado uma boa adequação aos dados, apresentou uma pequena retração nos primeiros diâmetros. Já os perfis obtidos por Max \& Burkhart e Schöepfer apresentaram a melhor representatividade do perfil médio do fuste. 

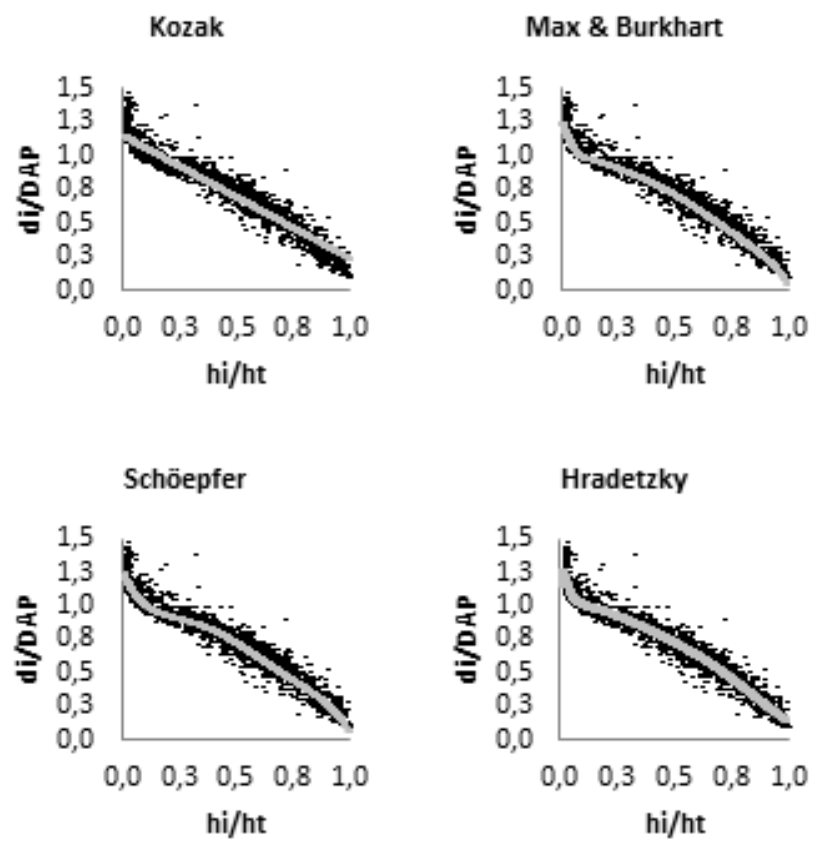

Figura 2. Perfil do fuste para estimar diâmetros ao longo do fuste de Pinus elliottii.

A validação da função de melhor desempenho, nesse caso a de Max \& Burkhart, apresentou resultado satisfatório com Syx \% de 6,14 \% e $R_{a j}^{2}$. de 0,99, resultado superior ao encontrado no próprio ajuste, demonstrando desempenho adequado na aplicabilidade da função. 0 teste Qui-quadrado resultou em um valor calculado de 38,02 , sendo o valor tabelado a $95 \%$ de probabilidade equivalente a 54,57, demonstrando que há aderência dos dados em relação à equação ajustada.

Na Figura 3 são apresentados os resultados da análise gráfica dos resíduos e a linha média de ajuste em relação aos dados da validação do modelo de Max \& Burkhart.É possível observar que a base do perfil do tronco estimado pelo modelo de equação de Max \& Burkhart, se assemelha ao sólido de revolução neilóide, seguido pelo formato de parabolóide e posteriormente por conóide.

Os diâmetros de melhores estimativas estão entre $5 \mathrm{~cm}$ e $25 \mathrm{~cm}$, sendo que existe uma leve superestimação nos diâmetros acima de $25 \mathrm{~cm}$ e uma alta amplitude de variação entre a estimativa dos diâmetros abaixo de $5 \mathrm{~cm}$. Se observa que a linha média de ajuste da equação obtida em relação aos dados, se encontra no centro da distribuição dos resíduos dos diâmetros das árvores separadas para a validação, indicando que o ajuste selecionado representa adequadamente todo o tronco.

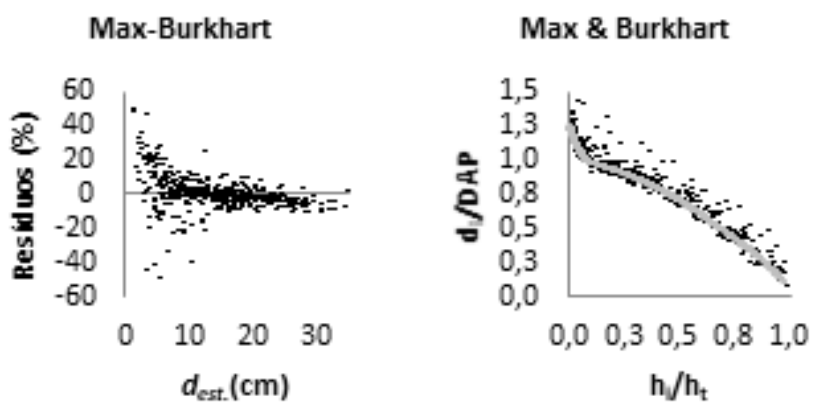

Figura 3. Distribuição dos Resíduos em função dos diâmetros e perfil do fuste da validação do melhor ajuste.

\section{DISCUSSÃO}

Para estudar o comportamento da forma dos fustes de árvores de Pinus elliotii provenientes de plantios localizados na região de Caçador, estado de Santa Catarina, com idades de 10, 18 e 27 anos, Téo et al. (2013) ajustaram os modelos de afilamento de Kozak et al., Schöepfer e Hradetzky. Os autores obtiveram valores de $R_{a j}^{2}$. e $S_{y x} \%$ variando respectivamente, entre 0,95 - 0,98 e 6,06 - 13,08 $\%$. Também se constatou que, semelhante ao ocorrido nesse trabalho, com exceção do modelo proposto por Max \& Burkhart, que não foi ajustado pelos autores, o modelo de Hradetzky apresentou superioridade aos de Schöepfer e Kozak et al. Assim, é possível observar que embora os melhores modelos sejam diferentes, comparados aos resultados do presente estudo, os valores dos indicadores estatísticos de regressão foram são semelhantes.

Ao estudar as relações dendrométricas em plantios de Pinus sp. na região norte do estado de Minas Gerais, Araújo et al. (2012) ajustaram 8 modelos de função de afilamento. Os ajustes apresentaram $S_{y x} \%$ de até $15 \%$ e $R_{a j}^{2}$. maiores que 0,90 , semelhante a esse trabalho, o modelo proposto por Max \& Burkhart apresentou o menor $S_{y x} \%$ e o maior $R_{a j,}^{2}$, seguido pelo modelo segmentado de Parresol e pelo não-segmentado de Hradetzky, cujas potências selecionadas foram 0,$7 ; 7 ; 0,005$ e 4 , o que confirma o bom desempenho do modelo de Max \& Burkhart para espécies do gênero Pinus sp., assim como também a desconexão entre a procedência dos indivíduos e a precisão dos ajustes.

Rojo et al., (2005) ajustou 31 modelos de funções de afilamento, classificados em 3 grupos (simples, segmentados e de forma variada) para plantios equiâneos de Pinus pinaster Ait. na região da Galícia, noroeste da Espanha. O modelo proposto por Max e Burkhart apresentou bom desempenho, sendo classificado como o 
melhor modelo do grupo de segmentados e o quinto modelo no geral, com $R_{a j}^{2}$. de 0,9873.

Tiarks e Clark Junior (1999) estudaram a validações de equações de afilamento ajustados para plantios de Pinus elliottii em florestas manejadas com predominância de Pinus elliottii, com idades entre 18 e 62 anos, localizadas no De Soto National Forest, estado do Mississippi e florestas manejadas com predominância de Pinus taeda, com idades entre 21 e 87 anos, localizadas no Kisatchie National Forest, estado de Louisiana e Davey Crockett National Forest, estado do Texas, todos localizados nos EUA, os autores relatam que por mais que existam diversos modelos de função de afilamento propostos por vários autores, alguns mais e outros menos flexíveis, a influência da idade e dimensão dos indivíduos são os fatores de maior influência na forma do fuste das espécies estudadas.

Max \& Burkhart (1976) relatam a espécie e a genética como fatores de influência da forma do fuste, enquanto que Amateis \& Burkhart (1987) afirmam que a origem do povoamento florestal, tratamentos silviculturais e nutrição dos indivíduos também afetam severamente a forma do fuste das árvores. Em vista disso e em comparação com os resultados obtidos para povoamentos de Pinus sp, pelos diferentes autores anteriormente citados, observa-se que no presente estudo, os fatores ligados a regeneração natural, como a maior variação genética, idade e espaçamento entre os indivíduos do povoamento estudado, embora possam afetar a forma do fuste, não apresentaram influência negativa sobre a precisão dos ajustes realizados.

\section{CONCLUSÕES}

O modelo de Max \& Burkhart é o mais acurado para estimar os diâmetros ao longo do fuste de Pinus elliotti sobre condições de regeneração, o que permite descrever a forma dos troncos e a possibilidade da determinação de sortimentos para múltiplos produtos da madeira. Quanto à precisão, conclui-se que a procedência dos indivíduos não afetou a precisão das equações ajustadas.

Portanto a utilização de modelos de afilamento para realizar a estimativa de sortimentos para povoamentos oriundos de regeneração natural de Pinus sp. é viável e de grande ajuda para planejamento e otimização da produção.

\section{REFERÊNCIAS}

AMATEIS, R.L.; BURKHART, H.E. Tree volume and taper of loblolly pine varies by stand origin. Southern Journal of Applied Forestry, v.11, n.4, p.185-189, 1987.
ARAÚJO, E.J.G. et al. Relações dendrométricas em fragmentos de povoamentos de pinus, em Minas Gerais. Pesquisa Florestal Brasileira, v.32, n.72, p.355, 2012.

CARVALHO, S.D.P. et al. Modelagem não linear mista para descrever o afilamento de árvores clonais de Eucalyptus sp. Scientia Forestalis, v.42, n.104, p.605-614, 2014.

FERRARI, L.S. et al. Equações de volume para condução da regeneração natural de Pinus elliottii Engelm. Revista Espacios, v.38, n.42, p.19-31, 2017.

FOX, T.R. et al. The development of pine plantation silviculture in the southern United States. Journal of Forestry, v.105, n.7, p.337347, 2007.

HORLE, D.C. et al. Modelagem não linear do perfil longitudinal de fustes de Pinus oocarpa. Cerne, v.16, n.2, p.177-184, 2010.

HRADETZKY, J. Analysis und interpretation statistisher abränger keiten (Biometrische Beiträge zu aktuellen forschungs projekten). Baden-Württemberg Mitteilungen der FVA, 1976.

KOHLER, S.V. et al. Modelos de Afilamento para Pinus taeda por Classes de Idade. Floresta e Ambiente, v.20, n.4, p.470-479, 2013.

ALVARES, C.A. et al. Köppen's climate classification map for Brazil. Meteorologische Zeitschrift, v.22, n.6, p.711-728, 2013

KOZAK, A. et al. Taper functions and their application in forest inventory. Forest Chronicle, v.45, n.4, p.278-283, 1969.

GONZATTI, F. et al. Florística e aspectos ecológicos de licófitas e samambaias do litoral médio do Rio Grande do Sul, Brasil. Revista Brasileira de Biociências. v.12, n.4, p.215-225, 2014.

MACFARLANE, D.W.; WEISKITTEL, A.R. A new method for capturing stem taper variation for trees of diverse morphological types. Canadian Journal of Forest Research, v.46, n.6, p.804-815, 2016.

MAX, T.A.; BURKHART, H.E. Segmented polynomial regression applied to taper equations. Forest Science, v.22, n.3, p.283-289, 1976.

MARTIN, S.S. Análise física e econômica de dois sistemas de manejo de Pinus elliottii Engelm em duas áreas do sul do Brasil. 2017. 9p. (Trabalho de conclusão de curso). Disponível em: http://calvados.c3sl.ufpr.br/handle/1884/45593. Acesso em $\underline{07 / 11 / 2017}$

MENDONÇA, A.R. et al. Avaliação de funções de afilamento visando a otimização de fustes de Eucalyptus sp. para multiprodutos. Cerne, v.13, n.1, p.71-82, 2007.

MÔRA, R. et al. Análise de diferentes formas de ajuste de funções de afilamento. Scientia Forestalis, v.42, n.102, p.237-249, 2014.

NAKAJIMA, N.Y. et al. Comparação da precisão entre os métodos de amostragem linha e parcela circular concêntrica em povoamentos de Pinus elliottii. Revista Acadêmica: Ciência Animal, v.9, n.2, p.131-139, 2011. 
ROJO, A. et al. Stem taper functions for maritime pine (Pinus pinaster Ait.) in Galicia (Northwestern Spain). European Journal of Forest Research, v.124, n.3, p.177-186, 2005.

SCHÖEPFER, W. Automatisierrung des massen, Sorten und Wertberechnung stenender Waldbestande Schriftenreihe, Bad. Koblenz: Wurtt-Forstl. v.21, 1966.

SOUZA, C.A.M. et al. Avaliação de modelos de afilamento nãosegmentados na estimação da altura e volume comercial de Eucalyptus sp. Ciência Florestal, v.18, n.3, p.387-399, 2008.

TÉO, S.J. et al. Modelos de Afilamento para Pinus elliottii em Diferentes Idades, na Região de Caçador, SC. Floresta, v.43, n.3, p.439-452, 2013.

TIARKS, A.E.; CLARK JUNIOR, V. Validation of volume and taper equations for Loblolly short leaf and Slash Pine. Tenth Biennial Southern Silvicultural Research Conference, p.16-18, 1999.

VASQUEZ, A.G. et al. Uma síntese da contribuição do gênero Pinus para o desenvolvimento sustentável no sul do Brasil. Floresta, v.37, n.3, p.445-450, 2007.

YOSHITANI JUNIOR, M. et al. funções de afilamento para plantios desbastados de Pinus taeda. Floresta, v.42, n.1, p.169-176, 2012. 